\title{
BIOSORCIÓN DE Pb (II) DE AGUAS RESIDUALES DE MINA USANDO EL MARLO DE MAÍZ (Zea mays)
}

\author{
Franklin Oré Jiménez a*; Carmencita Lavado Meza b, Salvador Bendezú Montes c
}

\begin{abstract}
RESUMEN
Se investigó la remoción de los iones de plomo desde soluciones acuosas utilizando marlo de maíz (MRLZ), mediante un sistema batch, en función del tiempo de contacto, dosis del biosorbente, $\mathrm{pH}$ inicial de la solución y concentración inicial de la solución de $\mathrm{Pb}$ (II). Para determinar los parámetros cinéticos, los datos experimentales fueron correlacionados utilizando tres modelos matemáticos (Langergren, Pseudo Segundo Orden y Elovich). Los datos experimentales de equilibrio fueron correlacionados utilizando dos modelos de dos parámetros: Langmuir y Freundlich; el modelo que mejor se ajustó fue el de Langmuir. Se estudió la influencia de los iones competitivos observando un decrecimiento en el porcentaje de biosorción de $\mathrm{Pb}(\mathrm{II})$. El MRLZ posee una capacidad máxima de biosorción en monocapa de $7,9 \mathrm{mg} / \mathrm{g}$. Se logró remover el $97 \%$ del $\mathrm{Pb}$ (II) contenido en las aguas residuales de mina. Palabras clave: Biosorbente, aguas residuales de mina, cinética, equilibrio de biosorción.

\section{BIOSORPTION OF LEAD PB (II) FROM RESIDUAL WATERS OF MINE BY CORNCOB (Zea mays)}

\begin{abstract}
Removing lead ions was investigated from aqueous solutions using corncob (MRLZ) by a batch system, depending on the contact time, dose biosorbent, and initial $\mathrm{pH}$ of the solution and initial concentration of the solution of $\mathrm{Pb}$ (II). To determine the kinetic parameters, experimental data were correlated using three mathematical models (Peudo primer orden, Pseudo second order and Elovich). The experimental equilibrium data were correlated using two-parameter models: Langmuir and Freundlich; model that best adjusted was the Langmuir. The influence of competing ions observing a decrease in the percentage of biosorption of $\mathrm{Pb}$ (II) was studied. The MRLZ has a maximum capacity of monolayer biosorption 7,9 $\mathrm{mg} / \mathrm{g}$. It was possible to remove $97 \%$ of the $\mathrm{Pb}$ (II) content in mine wastewater.
\end{abstract}

Keywords: Biosorbent, mine wastewater, kinetics, equilibrium.

\section{INTRODUCCIÓN}

La contaminación del agua por metales pesados es uno de los problemas ambientales más severos, debido a su alta toxicidad y su capacidad de bioacumulación en organismos vivos ${ }^{1}$. El incremento de la contaminación de aguas por metales pesados se debe a que éstos no son química ni biológicamente degradables ${ }^{2}$. El plomo es un metal tóxico que tiene la capacidad de biomagnificarse en el medio ambiente y causar daños en la salud, como la anemia, la hepatitis y la encefalopatía ${ }^{3}$. Los efluentes residuales provenientes de la minería, fábrica de pinturas y baterías, contienen altas concentraciones de iones $\mathrm{Pb}$ (II) ${ }^{4}$. Los métodos convencionales utilizados para la remoción de $\mathrm{Pb}$ (II) y otros metales pesados son: filtración con membrana,

$\mathrm{a}^{*}$ Facultad de Ingeniería y Arquitectura, Universidad Alas Peruanas, av. Coronel Parra km 5, Pilcomayo, Huancayo, e-mail, frankore.ingenieroambiental@gmail.com

b Facultad de Agronomía, c Facultad de Ingeniería Química, Universidad Nacional del Centro del Perú, Av. Mariscal castilla km 5, El Tambo, Huancayo. 
precipitación química, intercambio iónico y adsorción con carbón activado; estas tecnologías muchas veces resulta no ser utilizadas por el alto costo de operación.

Entre estas técnicas disponibles hoy en día, la biosorción ha demostrado ser eficiente y ventajosa debido a que el material biosorbente; es de fácil manejo en el tratamiento, puede retener el metal en presencia de otros cationes, el material biosorbente se puede regenerar mediante la desorción del metal, y sobre todo se puede encontrar en abundancia en la naturaleza y son de bajo costo. Para este trabajo se seleccionó como biosorbente al marlo de maíz (Zea mays) que es un material de desecho, producto de la agroindustria, y se encuentra en abundancia en la región. El presente estudio tuvo por objetivo caracterizar al biosorbente, realizar pruebas de biosorción de $\mathrm{Pb}$ (II) desde soluciones acuosas y estudiar las variables de dosis del biosorbente, cinética, concentración inicial de $\mathrm{Pb}$ (II) y pH, así como también correlacionar los datos experimentales de equilibrio y cinética de biosorción con los modelos matemáticos, estudiar la posibilidad de recuperar el metal y su aplicación en el tratamiento de un efluente minero.

\section{PARTE EXPERIMENTAL}

\section{Preparación del biosorbente y la solución de $\mathbf{P b}$.}

Para la eliminación de impurezas se lavó el marlo de maíz con abundante agua destilada. Luego el biosorbente fue transferido a un horno y secado a $60^{\circ} \mathrm{C}$ por 24 horas. Las muestras secas fueron molidas y tamizadas a malla $50 \mu \mathrm{m}$. Las soluciones de $\mathrm{Pb}$ (II) fueron preparadas disolviendo sal de nitrato de plomo, $\mathrm{Pb}\left(\mathrm{NO}_{3}\right)_{2}$, (Scharlau, Lead (II) grade ACS, 99,5\%) en agua desionizada.

\section{Caracterización del biosorbente.}

El área superficial se obtuvo mediante adsorción de $\mathrm{N}_{2}$ a $77 \mathrm{~K}$ en un equipo de adsorción Micromeritics, modelo Gemini VII (serie t).Para la determinación del área superficial y volumen de microporo se usó las ecuaciones de BET (Brunauer- Emmett-Teller) y DR (Dubinin-Radushkevich). Los grupos funcionales en el biosorbente se estudiaron por espectroscopía infrarroja con transformadas de Fourier (FTIR) en un Espectrofotómetro SHIMADZU FTIR- 8700; para ello se empleó 0,002 gr de biosorbente y 0,2 gr de KBr; las muestras se homogenizaron y pulverizaron en un mortero de ágata y secadas por $48 \mathrm{~h}$ a $60 \mathrm{oC}$ en un horno para luego ser analizadas en un rango espectral de 4000 a $400 \mathrm{~cm}^{-1}$. La determinación de los grupos ácidos, se llevó a cabo siguiendo el método Boehm 5; se basa en el hecho de que una base fuerte, como el $\mathrm{NaOH}$, neutraliza los centros ácidos presentes

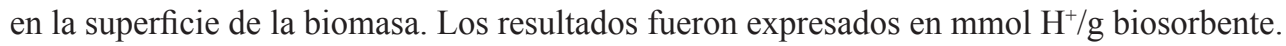

\section{Experimentos de biosorción}

La sorción de los iones $\mathrm{Pb}$ (II) desde soluciones acuosas se llevó a cabo en sistema batch, utilizando $100 \mathrm{~mL}$ de muestra de solución de $\mathrm{Pb}$ (II); los parámetros de velocidad de agitación $(150 \mathrm{rpm})$ y temperatura $\left(20^{\circ} \mathrm{C}\right)$ se mantuvieron constantes. Se estableció la dosis del biosorbente utilizando cantidades diferentes del marlo de maíz (1, 2, 4, 8, 12 y 16 g/L). Para determinar el equilibrio cinético se trabajó a diferentes intervalos de tiempo en un rango de 1-180 min. El equilibrio de sorción se evaluó a diferentes concentraciones iniciales del adsorbato en un rango de $2-50 \mathrm{mg} / \mathrm{L}$ de $\mathrm{Pb}$ (II). El estudio de la influencia del $\mathrm{pH}$ se realizó ajustando $\mathrm{pH}$ de la solución inicial en un rango de 2-6 utilizando soluciones de $\mathrm{NaOH}$ y $\mathrm{HNO}_{3}$ a 0,1 M. En cada uno de los casos luego del experimento, las fases se separaron 
por filtración utilizando papel filtro Wattman $\mathrm{N}^{\circ} 40$. La concentración final del plomo en la solución se determinó por absorción atómica con un espectrofotómetro marca SHIMADZU modelo AAS 6800 a una longitud de onda de 283,3nm.

La cantidad de iones de plomo retenidos por el biosorbente $\left(\mathrm{q}_{\mathrm{e}}, \mathrm{mg} / \mathrm{g}\right)$ y el porcentaje de biosorción se calcularon mediante las siguientes ecuaciones, respectivamente:

$$
q_{e}=\frac{\left(C_{0}-C_{e}\right)}{M} \times V \quad ; \quad \% R=\frac{C_{0}-C_{e}}{C_{0}} \times 100 \%
$$

Donde $\mathrm{C}_{0} \mathrm{y} \mathrm{C}_{\mathrm{e}}$ son las concentraciones inicial y final de la solución de los iones plomo $(\mathrm{mg} / \mathrm{L})$ antes y después de la biosorción, respectivamente; $M$ es la masa del biosorbente (g), y $V$ es el volumen de la solución (L).

Caracterización del biosorbente

\section{RESULTADOS Y DISCUSIÓN}

Análisis próximo: En la tabla 1 se muestra los resultados del análisis próximo del MRLZ.

Tabla 1. Análisis próximo del marlo de maíz (MRLZ).

\begin{tabular}{lr}
\hline & $\%$ peso \\
\hline Humedad & 13,8 \\
Material volátil & 72,8 \\
Cenizas & 2,91 \\
Carbón fijo & 10,49 \\
\hline
\end{tabular}

Análisis textural y determinación de grupos ácidos. El análisis de las propiedades texturales se realizó a través de la determinación de las isotermas de adsorción de $\mathrm{N}_{2}$ en el sistema sólido-gas y utilizando las ecuaciones BET. Para la determinación de los grupos ácidos se empleó el método Boehm. La tabla 2 muestra los resultados de estos análisis.

Tabla 2. Caracterización textural y contenido de grupos ácidos del MRLZ

\begin{tabular}{|c|c|c|c|c|c|}
\hline \multirow{2}{*}{ Biomasa } & \multicolumn{4}{|c|}{ Estructura del poro } & \multirow{2}{*}{$\begin{array}{c}\begin{array}{c}\text { Grupos ácidos } \\
\text { (Boehm) }\end{array} \\
\mathrm{mmol} \mathrm{H}^{+} / \mathrm{g} \\
\mathrm{MRLZ}\end{array}$} \\
\hline & $\begin{array}{c}\text { Área } \\
\text { superficial } \\
\text { BET }\left(\mathrm{m}^{2} / \mathrm{g}\right)\end{array}$ & $\begin{array}{c}\text { Diámetro } \\
\text { de poro } \\
\text { nm }\end{array}$ & $\begin{array}{c}V_{m} \\
\left(\mathrm{~cm}^{3} / \mathrm{g}\right)\end{array}$ & $\begin{array}{c}V_{T} \\
\left(\mathrm{~cm}^{3} / \mathrm{g}\right)\end{array}$ & \\
\hline MRLZ & 2,1673 & 8,05 & $5,6 \times 10^{-5}$ & 0,02759 & 8,15 \\
\hline
\end{tabular}

Espectroscopía con transformadas de Fourier (FTIR). En la figura 1 se muestra el espectro del marlo de maíz donde se reconocen los siguientes grupos: la banda ancha a $3303,8 \mathrm{~cm}^{-1}$, es atribuida a los grupos hidroxilos (-OH); la banda a $2924,9 \mathrm{~cm}^{-1}$ fue asignada a los alcanos $\left(-\mathrm{CH}_{2}-\right)$; los picos intensos a 1726,2 y $1247,9 \mathrm{~cm}^{-1}$ es debido a la vibración de tensión y flexión en el plano del grupo de los ésteres (=C-O-C); la banda a $1620,8 \mathrm{~cm}^{-1}$ es característico de los alquenos $(\mathrm{C}=\mathrm{C})$; la banda a 1510,2 es asignado al grupo de los aminos (-NH-); el pico a $1420,4 \mathrm{~cm}^{-1}$ corresponde al estiramiento vibracional del ácido carboxílico (CO-OH) y la 
banda a 1043,4 $\mathrm{cm}^{-1}$ fue asignado a los anhídridos.

Todas las bandas mencionadas son características en materiales lignocelulósicos ${ }^{6}$.

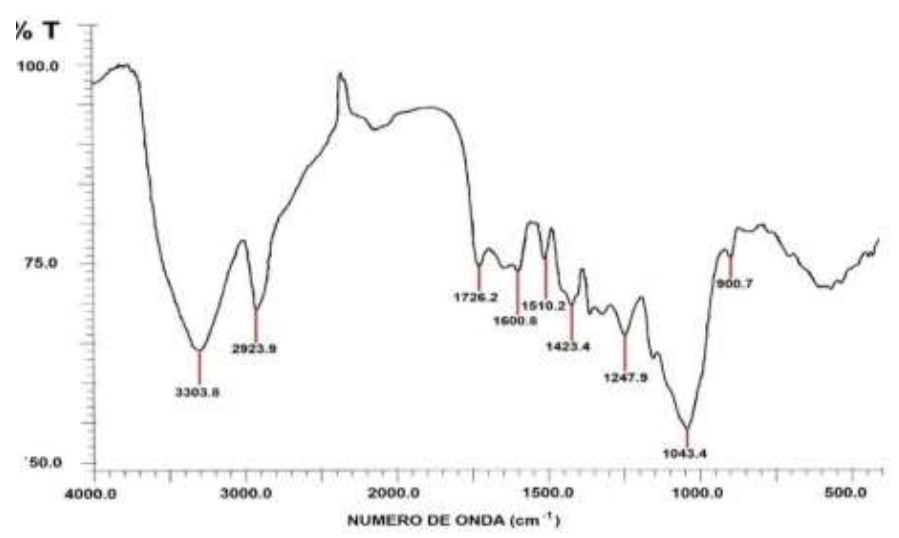

Figura 1. Espectros de FTIR del marlo de maíz antes del proceso de biosorción de $\mathrm{Pb}$ (II).

\section{Biosorción de $\mathbf{P b}(\mathrm{II})$}

Efecto de la concentración del biosorbente. El efecto de la concentración del biosorbente; fue estudiado usando $100 \mathrm{~mL}$ de una solución de $\mathrm{Pb}$ (II) y diferentes concentraciones del biosorbente; en la figura 2 se observa el incremento del \% de biosorción a medida que va aumentando la dosis del biosorbente. Este incremento está relacionado al aumento del área de contacto del sólido adsorbente con el adsorbato; de esta manera va aumentando el número de los sitios de biosorción disponibles; se observa también que un aumento de la concentración del biosorbente después de de $4 \mathrm{~g} / \mathrm{L}$ no provoca un cambio sustancial en el \% de biosorción, motivo por el cual la dosis que se usa en los ensayos próximos es de $4 \mathrm{~g} / \mathrm{L}$.

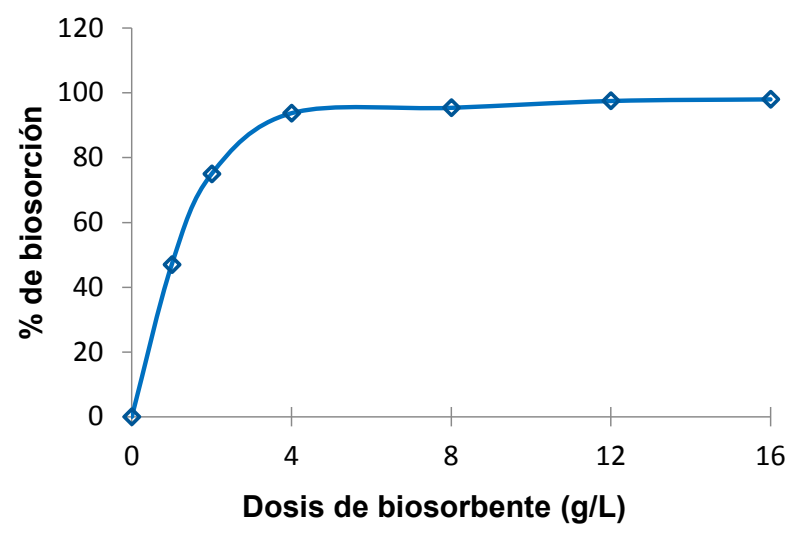

Figura 2. Relación del \% de biosorción con la concentración de biosorbente. 
Efecto de la concentración inicial de $\mathbf{P b}$ (II) sobre la biosorción. La tabla 3 ilustra la dependencia del proceso de biosorción de $\mathrm{Pb}(\mathrm{II})$ a diferentes concentraciones iniciales $(\mathrm{Co})$ de $\mathrm{Pb}(\mathrm{II})$ y $\mathrm{pH}$ inicial igual a 5. Se observa que la capacidad de biosorción (qe) del plomo es altamente dependiente de la concentración, conforme la concentración inicial se incrementa qe hace lo mismo hasta mantenerse casi constante luego de una concentración inicial de 30 $\mathrm{mg} / \mathrm{L}$, lo cual se explicaría por la saturación de los centros activos de adsorción.

Isotermas de adsorción: Las isotermas de sorción, representa las cantidades de $\mathrm{Pb}$ (II) $(\mathrm{mg})$ retenido por el biosorbente ( $\mathrm{g}$ ) y describen la manera como interactúa el adsorbato con la superficie del material adsorbente. La isoterma fue obtenida graficando la capacidad de adsorción (qe) expresado en $\mathrm{mg}$ de $\mathrm{Pb}(\mathrm{II})$ )/g de biomasa versus la concentración del $\mathrm{Pb}(\mathrm{II})$ en equilibrio $\left(\mathrm{C}_{\mathrm{e}}\right)$ y se muestra en la figura 4 donde se observa que la curvatura inicial de la isoterma se va separando a medida que se incrementa el valor de $\mathrm{C}_{\mathrm{e}}$, lo que estaría indicando que las moléculas de $\mathrm{Pb}(\mathrm{II})$ tienen más dificultad para encontrar un sitio activo de adsorción, la capacidad de adsorción; se incrementa hasta casi alcanzar una condición estable de 7,5 mg $\mathrm{Pb}(\mathrm{II}) / \mathrm{g}$.

Correlación de los valores experimentales con los modelos de isotermas. Los datos experimentales fueron correlacionados con dos modelos de isotermas:

El modelo de Langmuir asume una sorción del soluto en monocapa con una energía de sorción homogénea ${ }^{7}$. Este modelo es expresado mediante la siguiente ecuación lineal (1a) y no lineal (2a).

$$
q e=q_{\max } \frac{K_{L} \cdot C_{e}}{1+K_{L} \cdot C_{e}} \quad \text { (1a) } \quad ; \quad \frac{C e}{q_{e}}=\frac{1}{K_{L} \cdot q_{\max }}+\frac{C e}{q_{\max }}
$$

Dónde: qe y $q_{\max }(\mathrm{mg} / \mathrm{g})$ es la capacidad de sorción en el equilibrio y en monocapa; $K_{L}$, es una constante que representa la afinidad entre el adsorbato y el adsorbente, $C_{e}(\mathrm{mg} / \mathrm{L})$, es la concentración del adsorbato en el equilibro. Los valores de $q_{\max }$, y $K_{L}$ se hallaron de las ecuación linealizada 2a, luego de graficar $C e / q e$ versus $C_{e}$ (figura 3a).

El modelo de Freundlich es una expresión empírica que asume la sorción del soluto en multicapas, considerando que las energías de sorción son heterogéneas ${ }^{8}$. El modelo de Freundlich es expresado mediante la siguiente ecuación no lineal 1b, y lineal $2 \mathrm{~b}$.

$$
q_{e}=K_{F} \cdot C_{e}^{1 / n}(1 \mathrm{~b}) \quad ; \quad \log q_{e}=\log K_{F}+\left(\frac{1}{n}\right) \cdot \log C e
$$

Donde $K_{F}(\mathrm{~L} / \mathrm{g})$ es una constante de la adsorción en equilibrio y n es una constante característica asociada al grado de favorabilidad de la adsorción. Estos valores se hallaron, de la ecuación lineal 2b; luego de graficar $\log q e$ versus $\log C e$ (figura $3 \mathrm{~b}$ ). 

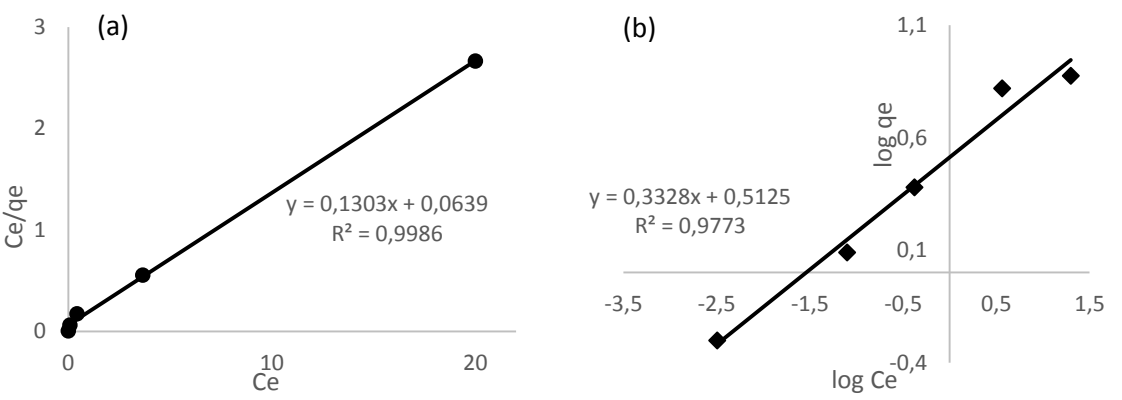

Figura 3. Correlación de los datos experimentales empleando (a) el modelo de Langmuir y , (b) el modelo de Freundlich para la biomasa MRLZ.

En la figura 3 se puede observar las correlaciones de los modelos de Langmuir (a), y Freundlich (b). En la tabla 3 se puede observar las constantes de ambos modelos, examinando las correlaciones se observa que los valores experimentales se ajustan mucho mejor al modelo de Langmuir con un valor de $\mathrm{R}^{2}=0.9986$, y una $\mathrm{K}_{\mathrm{L}}=2,0391$ que predice una sorción en monocapa en centros activos energéticamente homogéneos.

Tabla 3. Parámetros de los modelos de las isotermas de adsorción

\begin{tabular}{ccccccc}
\hline Biomasa & \multicolumn{2}{c}{ Constantes de Langmuir } & \multicolumn{5}{c}{ Constantes de } \\
& \multicolumn{5}{c}{ Freundlich } \\
\cline { 2 - 7 } & $\mathbf{q} \max (\mathbf{m g} / \mathbf{L})$ & $\mathbf{K}_{\mathbf{L}}(\mathbf{L} / \mathbf{m g})$ & $\mathbf{R}^{\mathbf{2}}$ & $\mathbf{K}_{\mathbf{F}}$ & $\mathbf{n}$ & $\mathbf{R}^{\mathbf{2}}$ \\
MRLZ & 7,6745 & 2,0391 & 0,9986 & 1,6695 & 3,005 & 0,9773 \\
\hline
\end{tabular}

En la figura 4 se muestra la forma de la isoterma, la cual indica la alta afinidad del adsorbato por el adsorbente debido a que no existe competición por los sitios de adsorción entre el solvente $\left(\mathrm{H}_{2} \mathrm{O}\right)$ y soluto $\mathrm{Pb}(\mathrm{II})$. Las isotermas de Langmuir y Freundlich (figura 5), se graficaron con los datos calculados a partir de la ecuación lineal de cada modelo; reemplazando las constantes calculadas de la ecuación no lineal en cada caso.

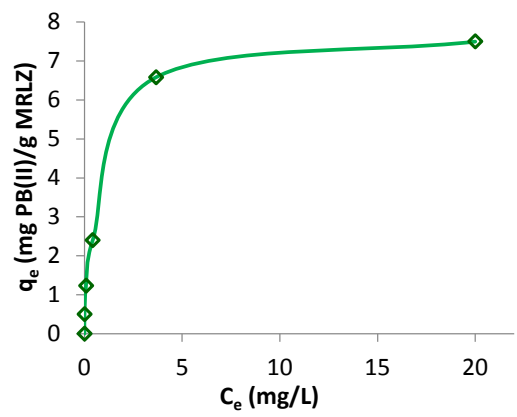

Figura 4. Comparación de la isoterma experimental correlacionadas a los modelos de Langmuir y Freundlich.

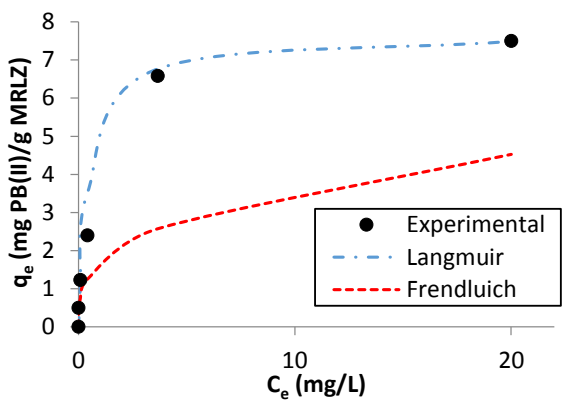

Figura 5. Isotermas para la adsorción de $\mathrm{Pb}$ (II) utilizando marlo de maíz, $\mathrm{T}=20^{\circ} \mathrm{C}, \mathrm{pH}$ 
Cinética de biosorción. En la figura 6 se muestra el efecto del tiempo de contacto adsorbatoadsorbente sobre la biosorción de $\mathrm{Pb}$ (II) en la biomasa (MRLZ). Se observa una sorción rápida; durante los 10 primeros minutos y se alcanza el equilibrio aproximadamente en 90 minutos; el valor de la capacidad de adsorción (qe) aumenta con el incremento del tiempo de contacto; una vez alcanzado el equilibrio, las curvas de ambas figuras se hacen constantes. Un comportamiento similar fue reportado por Akar et al., quienes trabajaron con cascaras de coco alcanzando un equilibrio a los $80 \min ^{9}$.

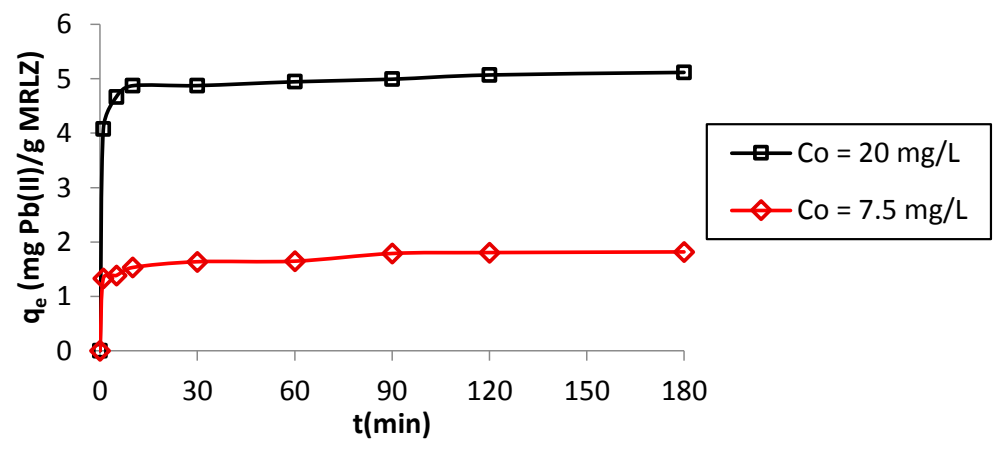

Figura 6. Cinética de biosorción del marlo de maíz.

Correlación de los datos cinéticos: En el proceso de biosorción los parámetros cinéticos proporcionan importante información para el diseño y modelamiento del proceso. Los datos experimentales obtenidos fueron correlacionados en base a tres modelos diferentes: La ecuación de Pseudo-Primer Orden (ecuación de Lagergren) describe la adsorción en sistemas sólido-líquido basado en la capacidad de sorción de los sólidos ${ }^{10}$. Esta dada mediante la siguiente ecuación:

$$
q_{t}=q_{e}\left(1-e^{-k_{1} t}\right)
$$

Donde: $q_{t} \mathrm{y} q_{e}(\mathrm{mg} / \mathrm{g})$ en cualquier tiempo $(t)$ y en el equilibrio $(e)$ y $k_{1}$ es la constante de velocidad de primer orden $\left(\mathrm{min}^{-1}\right)$. Este modelo permite obtener la constante de velocidad de Pseudo-Primero Orden $\left(\mathrm{k}_{1}\right)$, considerando que la velocidad de adsorción depende únicamente de la concentración inicial del adsorbato.

El modelo de Pseudo-Segundo Orden describe la cinética de quimisorción de soluciones líquidas y asume que los iones metálicos se adsorben en dos sitios distintos de sorción de la superficie adsorbente ${ }^{11}$.

$$
q_{t}=q_{e} \frac{q_{e} \cdot k_{2} \cdot t}{1+q_{e} \cdot k_{2} \cdot t}
$$


Donde $k_{2}$ es la constaste de velocidad de segundo orden $\left(\mathrm{min}^{-1}\right)$, y se utiliza para calcular la velocidad inicial h (mg/g min); está dada mediante la siguiente ecuación.

$$
h=k_{2} \cdot q_{e}^{2}
$$

El modelo de Elovich describe el proceso de quimisorción ${ }^{12}$, determinada por.

$$
q=\frac{1}{b} \ln (a \cdot b \cdot t+1)
$$

Donde a representa la velocidad de quimisorción en tiempo cero y b está relacionado con la extensión de la cobertura de la superficie y la energía de activación para la adsorción.

Los valores de las constantes de velocidad de los modelos cinéticos a dos concentraciones iniciales se muestran en la tabla 4, al analizar los valores de $\mathrm{R}^{2}$ se determinó que el modelo de pseudo segundo orden es el que mejor se ajusta a los datos experimentales lo que permitiría establecer que el plomo se quimisorbe sobre la superficie del biosorbente.

\begin{tabular}{|c|c|c|c|c|c|c|c|c|c|}
\hline \multirow{2}{*}{$\begin{array}{c}\mathrm{C}_{0} \\
\mathrm{mg} / \mathrm{L}\end{array}$} & \multicolumn{2}{|c|}{$\begin{array}{c}\text { Pseudo primer } \\
\text { orden }\end{array}$} & \multicolumn{4}{|c|}{$\begin{array}{c}\text { Pseudo segundo } \\
\text { orden }\end{array}$} & \multicolumn{2}{|c|}{ Elovich } & \multirow[b]{2}{*}{$\mathbf{R}^{2}$} \\
\hline & $\begin{array}{c}\mathrm{K}_{1} \\
\min ^{-1}\end{array}$ & $\begin{array}{c}\text { qecal } \\
\text { mg g}^{-1}\end{array}$ & $\overline{\mathbf{R}^{2}}$ & $\begin{array}{c}\mathrm{K}_{2} \\
\mathrm{~g} / \mathrm{mg} \text { min }\end{array}$ & $\begin{array}{c}\mathbf{h} \\
\mathrm{mg} / \mathrm{g} \text { min }\end{array}$ & $\mathbf{R}^{2}$ & $\begin{array}{c}\mathrm{a} \\
\mathrm{mg} / \mathrm{g} \text { min }\end{array}$ & $\begin{array}{c}\text { b } \\
\mathrm{g} \mathrm{mg}^{-1}\end{array}$ & \\
\hline 7.5 & 1,6622 & 1,7340 & 0,9443 & 1,654 & 5,217 & 0,9643 & 101,9900 & 5,7322 & 0,9529 \\
\hline 20 & 1,7462 & 4,9346 & 0,9937 & 0,841 & 21,039 & 0,9980 & 101,9900 & 1,8161 & 0,8353 \\
\hline
\end{tabular}

Tabla 4. Constantes de los modelos cinéticos, $\mathrm{T}=20^{\circ} \mathrm{C}$

Mecanismo de biosorción: Se han propuesto muchos modelos cinéticos para elucidar el mecanismo de adsorción; éste depende de las características físicas y químicas del adsorbente; la técnica más usada para describir el mecanismo de adsorción es el uso del modelo de difusión intraparticular, el cual está dado mediante la siguiente ecuación:

$$
q_{t}=k_{d i} \cdot \sqrt{t}+c
$$

Donde, $k_{d i}$ es la constante de velocidad del modelo de difusión intraparticular y $c$ es una constante relacionada con el espesor de la capa límite del adsorbente. La gráfica mostrada en la figura 7 representa la correlación de los datos experimentales obtenidos con la biomasa con el modelo de difusión a dos concentraciones iniciales diferentes, como se puede observar presentan multilinealidad ${ }^{13}$. La primera porción lineal que se encuentra entre los valores de 0 - 1 min ${ }^{0.5}$ es atribuida al proceso de difusión a través de la solución del adsorbato hacia la superficie del adsorbente, los valores obtenidos indican que es un proceso bastante rápido; la segunda porción, identificada con un cambio de dirección en la curva a valores de 
1 y 3,5 $\min ^{0.5}$, describe el proceso de difusión interna; se puede observar que esta etapa se lleva a cabo gradualmente y la tercera porción, es atribuida a la etapa final de equilibrio que corresponde al proceso de sorción propiamente dicha ${ }^{14}$.

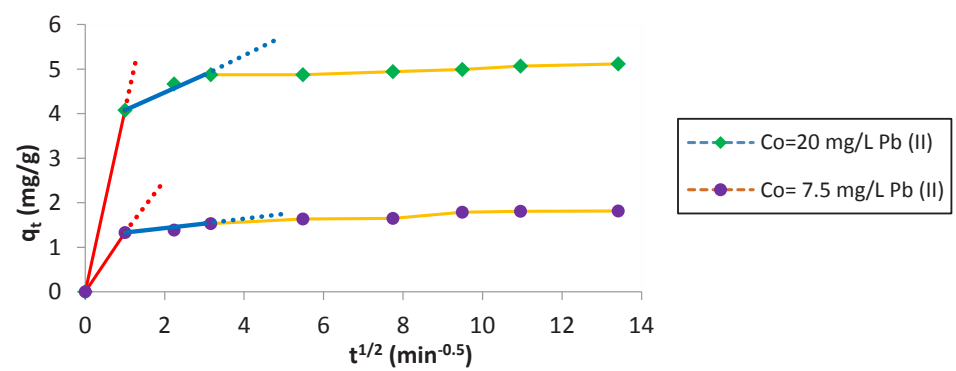

Tabla 7. Modelo cinético de difusión intraparticular para la sorción de Pb II.

Efecto del pH. Se puede considerar que el pH de la solución es el parámetro más importante que influye en la capacidad de adsorción de un adsorbato sobre la superficie de la biomasa, ya que influye grandemente en las interacciones electrostáticas superficiales entre la biomasa y las diferentes especies químicas del metal, puestas en contacto ${ }^{6}$. En la figura 9 se muestra la influencia del $\mathrm{pH}$ inicial de la solución sobre la remoción de $\mathrm{Pb}(\mathrm{II})$, a diferentes concentraciones iniciales del adsorbato. Para todas las concentraciones iniciales, está claro que para valores bajos de $\mathrm{pH}$ la capacidad de remoción es bastante baja, la capacidad de adsorción de $\mathrm{Pb}$ (II) se incrementa con el aumento del valor del pH, alcanzándose una máxima adsorción a pH 5; a valores mayores la capacidad de adsorción disminuye; no se realizó ensayos a pH mayores a 6 ya que a partir de este valor el $\mathrm{Pb}(\mathrm{II})$ empieza a precipitar.

Este comportamiento se puede explicar debido a que la pared celular del marlo de maíz contiene una cadena de grupos funcionales de ácidos débiles, grupos $\mathrm{OH}$ y carboxílicos característicos de materiales lignocelulósicos (esto se puede corroborar con los resultados de FTIR presentados anteriormente).

La biosorción se podría estar llevando a cabo debido a dos mecanismos: intercambio iónico e interacciones electrostáticas, (figura 8); a valores bajos de $\mathrm{pH}$ disminuye la remoción, ya que se tiene una mayor cantidad de iones $\mathrm{H}^{+}$los cuales estarían competiendo con los iones de $\mathrm{Pb}^{2+}$ por los sitios de intercambio en el sistema y a la vez se da una fuerza de repulsión; ya que la superficie se encuentra cargada positivamente.
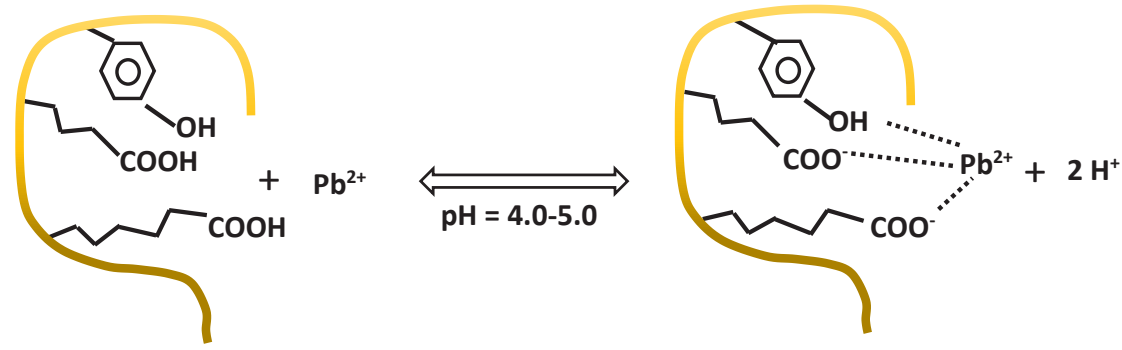

Figura 8. Mecanismo de biosorción de iones de $\mathrm{Pb}$ (II), con MRLZ. 
Cuando el pH se incrementa, más grupos funcionales están disociados y se convierten en provechosos enlazantes de iones $\mathrm{Pb}$ (II) esto debido a que hay menos competencia de iones $\mathrm{H}^{+}$ en la solución y por ende, hay una fuerza de atracción entre el metal $\mathrm{Pb}$ (II) con la superficie del biosorbente COO-. A pH mayores a 5.0, la especie dominante es $\mathrm{Pb}(\mathrm{OH})_{2} \mathrm{OH}$

y a $\mathrm{pH}<5,0$ las especies existentes son, $\mathrm{Pb}$ (II) ${ }^{15}$. A pH mayores a 5 la capacidad de sorción disminuye ya que el plomo precipita como $\mathrm{Pb}(\mathrm{OH})_{2}$; similar comportamiento fue reportado por Seyda et $a .^{6}$ quienes removieron $\mathrm{Pb}$ (II) con cáscaras de maní logrando una máxima remoción a $\mathrm{pH} 5$.

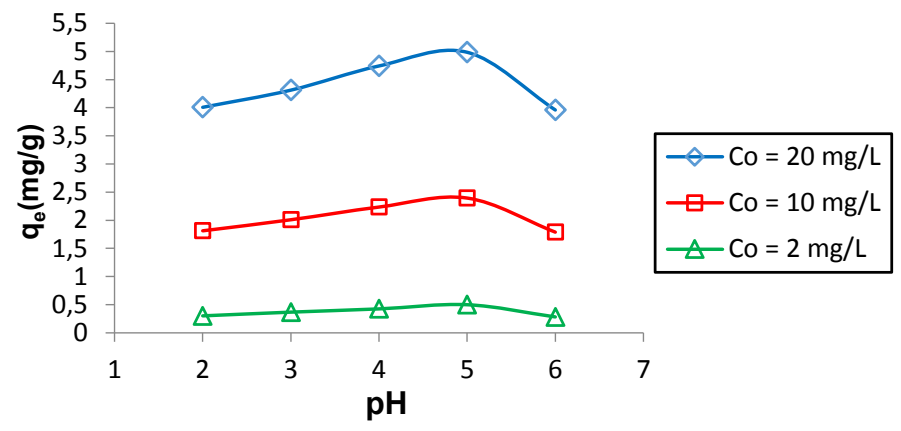

Figura 9. Efecto del $\mathrm{pH}$ en la biosorción de $\mathrm{Pb} \mathrm{II,} \mathrm{T}=20{ }^{\circ} \mathrm{C}$, dosis del biosorbente $4 \mathrm{~g} / \mathrm{L}$.

Experimentos de desorción. El proceso de desorción permite recobrar el metal adsorbido y la regeneración del biosorbente. La desorción del $\mathrm{Pb}(\mathrm{II})$ se desarrolló en sistema Bach con $100 \mathrm{~mL}$ de $\mathrm{HNO}_{3}$, a diferentes molaridades $(0,05 \mathrm{M}-0,3 \mathrm{M})$. Se puede observar en la figura 10 que la máxima desorción que se obtuvo fue de $96 \%$ con una concentración de $\mathrm{HNO}_{3}$ igual a 0,3 M. La desorción indica que el mecanismo de sorción se estaría llevando a cabo por intercambio iónico con los grupos funcionales presentes en la superficie del adsorbente, como ya se mencionó anteriormente. Estos resultados están de acorde con los reportados por Julio Vaghetti et al, ${ }^{16}$ donde realizó la desorción del plomo de cáscaras de pecanas y nuez con $\mathrm{HNO}_{3}$, alcanzando un $93,25 \%$ de desorción.

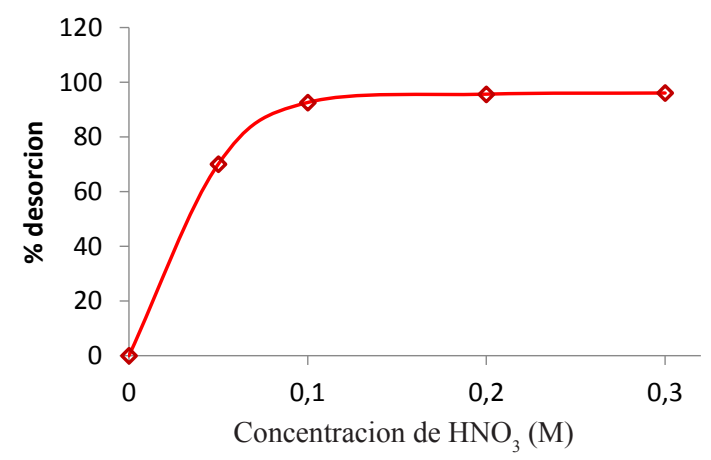

Figura 10. Desorción de $\mathrm{Pb}$ con diferentes concentraciones de $\mathrm{HNO}_{3}$ 
Tratamiento del efluente. Se realizó un análisis químico a la muestra, y se determinó que no solo contenía $\mathrm{Pb}$ II (7mg/L), sino también otros iones metálicos en abundancia: Mn (76 $\mathrm{mg} / \mathrm{L})$ y $\mathrm{Fe}(63 \mathrm{mg} / \mathrm{L})$, los cuales podrían ejercer una fuerza iónica que podría afectar la biosorción de plomo sobre el biosorbente de marlo de maíz (efecto selectivo o competitivo); para ello se preparó soluciones de los iones competitivos con concentraciones similares a la del efluente y se realizaron ensayos de biosorción en forma separada con cada una de ellas. En la tabla 5 se puede observar que el ion que ejerce mayor influencia en el proceso de biosorción es el Fe haciendo que el \% de remoción disminuya de 99,96 a 98,02.

Tabla 5. Influencia de los iones competitivos sobre la remoción de $\mathrm{Pb}$ (II).

\begin{tabular}{ll}
\hline Iones & \% R \\
\hline $\mathrm{Pb}^{2+}$ & 99,96 \\
$\mathrm{~Pb}^{2+} \mathrm{y} \mathrm{Fe}^{3+}$ & 98,02 \\
$\mathrm{~Pb}^{2+} \mathrm{y} \mathrm{Mn}^{2+}$ & 99,09 \\
$\mathrm{~Pb}^{2+}, \mathrm{Fe}^{3+} \mathrm{y} \mathrm{Mn}^{2+}$ & 97,36 \\
\hline
\end{tabular}

Se realizó el tratamiento de la muestra del agua residual de mina y se evaluó la capacidad de sorción de $\mathrm{Pb}$ (II) utilizando cantidades diferentes del biosorbente. La figura 11 muestra la relación entre la dosis del biosorbente y el \% de remoción; se puede observar que utilizando 0,4 g/L de biosorbente de marlo de maíz se puede remover el $97 \%$ del plomo, aun a pesar de la presencia de otros iones metálicos en abundancia ( $\mathrm{Mn}, \mathrm{Fe}, \mathrm{Zn}, \mathrm{Cu}$ ).

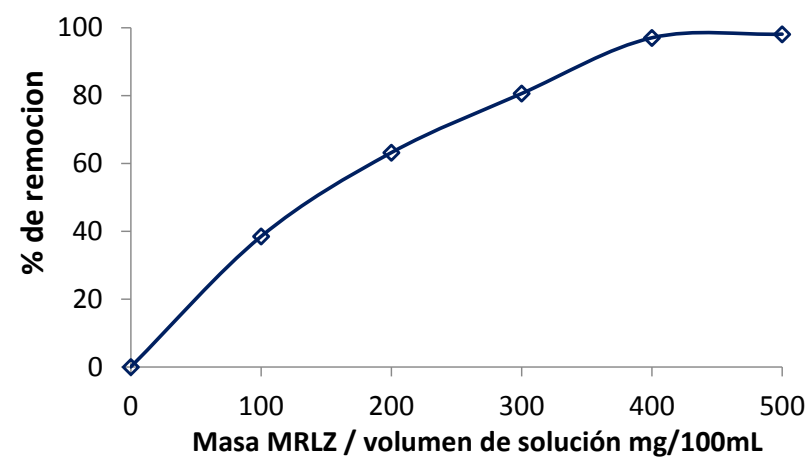

Figura 11. Estudio de la influencia de la relación masa de biosorbente/volumen de la solución sobre el \% de remoción. 


\section{CONCLUSIONES}

- Se caracterizó el biosorbente cuya área superficial es de $2,2232 \mathrm{~m}^{2} / \mathrm{g}$, cantidad de grupos ácidos superficiales $8,15 \mathrm{mmol}$ de $\mathrm{H}^{+}$y se determinó por FTIR la presencia de grupos funcionales como $\mathrm{OH}$ y grupos carboxílicos los cuales estarían favoreciendo el proceso de biosorción.

- De los ensayos de biosorción se determinó las condiciones óptimas para la remoción de $\mathrm{Pb}$ (II) las cuales fueron de $4 \mathrm{~g} / \mathrm{L}$ de biosorbente, tiempo de agitación de 90 minutos y $\mathrm{pH}$ 5,0 .

- La variable de mayor influencia sobre la capacidad de adsorción de $\mathrm{Pb}(\mathrm{II})$ fue el $\mathrm{pH}$. Se obtuvo la máxima remoción a un $\mathrm{pH}$ igual a 5. Se estableció que el proceso de biosorción se dio por intercambio iónico e interacción electrostática entre el adsorbato y la superficie del biosorbente MRLZ.

- El modelo de las isotermas que tuvo mejor ajuste fue el de Langmuir, el cual indica una biosorción en monocapa con un capacidad máxima de saturación de 7,49 mg/g, y para la cinética el modelo que mejor se ajustó a los datos experimentales fue el de pseudo segundo orden, lo que indicó que el adsorbato se quimisorbe sobre la superficie del biosorbente.

- Se logró remover el 97 \% de Pb II contenido en las aguas residuales de mina, lo cual indica un método eficiente y económico para el tratamiento de aguas residuales con $\mathrm{Pb}(\mathrm{II})$.

\section{AGRADECIMIENTOS}

Los autores agradecen el apoyo brindado para la elaboración de este trabajo de investigación, a la ingeniera Edith Solano del laboratorio de análisis instrumental de Universidad Nacional del Centro del Perú. Asimismo, se hace extensivo el agradecimiento al doctor Gino Picasso del laboratorio de investigación de fisicoquímica, facultad de ciencias, de la Universidad Nacional de Ingeniería por los análisis de área superficial realizados.

\section{REFERENCIAS}

1. Acosta I., M. G. Moctezuma-Zarate, J. F. Cárdenas y C. Gutiérrez. Bioadsorción de Cadmio (II) en Solución Acuosa por Biomasas Fúngicas. Información Tecnológica. 2007; (18): 9-14.

2. Cimino, G. And Caristi, C. Acute toxicity of heavy metals to aerobic digestion of waste cheese whey. Biological Wastes.1990; (33): 201-210.

3. L. Jarup, Hazards of heavy metal contamination, British Medical Bulletin. 2003; (68): $167-82$.

4. A. Ornek, M. Ozacar, I.A. Sengil, Adsorption of lead onto formaldehyde or sulphuric acid treated acornwaste: equilibrium and kinetic studies, Biochem. Eng. J. 2007; (37): 192-200.

5. Rochefort, A.; Wuest, J.D. Interaction of substituted aromatic compounds with graphene.Langmuir. 2009; (25): 210-215.

6. Tasar Seyda, Kaya Fatih, Ozer Ahmet. Biosorption of lead (II) ions from aqueous solution by peanut shells: Equilibrium, thermodynamic and kinetic studies. Journal of Environmental Chemical Engineering. 2014; (2): 1018-1026.

7. Seong-Rin, L.; Schoenung, J. M. Human health and ecological toxicity potentials due to heavy metal content in waste electronic devices with flat panel displays. Journal of 
Hazardous Materials. 2010 (177): 251-259.

8. Faur-Brasquet, C.; Kadirvelu,K.; Le Cloirec, P. Removal of metal ions from aqueous solution by adsorption onto activated carbon cloths: adsorption competition with organic matter. Carbon. 2002; (40): 2387-2392.

9. Akar Sibel Tunali. Biosorption potential of the waste biomaterial obtained from Cucumis melo for the removal of $\mathrm{Pb} 2+$ ions from aqueous media: Equilibrium, kinetic, thermodynamic and mechanism analisys. Chemical Engineering Journal. 2012; (185): 28-90.

10. Boparai H.K., Meera J., O'Carroll D. M. Kinetics and thermodynamics of cadmium ion removal by adsorption onto nano zerovalent iron particles. Journal of Hazardous Materials. 2011; (186): 458- 465.

11. Y.S. Ho, G. McKay, Kinetic models for the sorption of dye from aqueous solution by wood, Process Saf. Environ. Prot. 1998; (76): 183-191.

12. M.J.D., Low kinetics of chemisorption of gases on solids, Chem. Rev. 1960; (60): 267-31.

13. W.J. Weber Jr., J.C. Morris, Kinetics of adsorption on carbon from solution, J. Sanit. Eng. Div. Am. Soc. Civil Eng. 1963; (89): 31-59.

14. B. Guo, L. Hong, H.X. Jiang, Macroporous poly (calcium acrylate-divinylbenzene) bead-a selective orthophosphite sorbent, Ind. Eng. Chem. Res. 2003; (42).

15. Sekar M., Sakthi V., Rengaraj S.Kinetics and equilibrium study of lead (II) onto activated carbon prepared from coconut shell, Journal of Colloid and Interface Science 2004; (279): 307- 313.

16. Vaghetti, C., Lima, E., \& al, e. Pecan nutshell as biosorbent to remove $\mathrm{Cu}$ (II), $\mathrm{Mn}$ (II) and $\mathrm{Pb}$ (II) from aqueous solutions. Journal of Hazardous Materials. 2009; (162): 270-280. 